\title{
Targeting FOXM1
}

\author{
Stephen S. Myatt and Eric W.-F. Lam
}

We welcome the correspondence from Radhakrishnan and Gartel regarding our recent Review $^{1}$, which identifies the miscitation of a cell line used in their study ${ }^{2}$; however, this technicality does not distract from our inference that transformed cells are more sensitive to siomycin A-induced apoptosis than non-transformed counterparts.

We feel that the mechanism of action of thiazole antibiotics may be more complex than currently appreciated, and although we principally agree that the hypothesis described by the authors may in part account for the action of siomycin A, a complete model is currently lacking. In particular, we highlight the need to determine whether treatment of non-transformed cells with siomycin A also leads to a reduction in FOXM1 (also known as trident) expression or phosphorylation; this has not currently been described and might have important implications for the mechanism of action of siomycin A. The authors point to the existence of a positive feedback loop in vivo whereby siomycin A downregulates the expression of FOXM1 through inhibition of FOXM1 transcriptional activity, which qualifies the rationale that the effect of siomycin A upon FOXM1 protein level is not direct, but requires alteration in the expression of FOXM1, potentially through modulation of gene transcription. Moreover, after revisiting the original publication ${ }^{2}$ we feel that the physiological significance of a FOXM1 autoregulatory loop requires further validation and may potentially reflect a cell cycle effect of FOXM1 overexpression. Although absence of proof is not proof of absence, we remain open-minded regarding the mechanism of action of these compounds, and look forward to future advances in the field. In particular, thaizole-related compounds might provide a useful tool to explore the hypothesis, described in our Review, that cancer cells may become addicted to high levels of expression or activity of FOXM1.

Debate aside, we are pleased that the authors reiterate our conclusion that FOXM1 represents an exciting and promising target for therapeutic intervention in cancer. Indeed, recent evidence suggests that FOXM1 might have a role in the invasion and angiogenesis of pancreatic cancer ${ }^{3}$, and that FOXM1 might also be a crucial mediator of transcription factor AP-2 $\gamma$ (TFAP2C)-controlled oestrogen receptor $\alpha$ (ESR1) expression ${ }^{4}$, reinforcing the role of FOXM1 in hormonal response in patients with breast cancer. Thus, the gathering evidence suggests a scenario whereby enhanced FOXM1 expression or activity promotes three key features of cancer - cell transformation, tumour progression and metastasis - and that FOXM1 is a trident indeed.

Cancer Research UK Laboratories, Department of Oncology, MRC Cyclotron Building, Imperial College London, Hammersmith Hospital Campus, Du Cane Road, London, W12 ONN, UK Correspondence to E.L. e-mail:eric.lam@imperial.ac.uk

1. Myatt, S. S. \& Lam, E. W. The emerging roles of forkhead box (Fox) proteins in cancer. Nature Rev. Cancer 7, 847-859 (2007).

2. Radhakrishnan, S. K. et al. Identification of a chemical inhibitor of the oncogenic transcription factor forkhead box M1. Cancer Res. 66, 9731-9735 (2006).

3. Wang, Z., Banerjee, S., Kong, D., Li, Y. \& Sarkar, F. H. Down-regulation of Forkhead Box M1 transcription factor leads to the inhibition of invasion and angiogenesis of pancreatic cancer cells. Cancer Res. 67, 8293-8300 (2007).

4. Woodfield, G. W., Horan, A. D., Chen, Y. \& Weigel, R. J. TFAP2C controls hormone response in breast cancer cells through multiple pathways of estrogen signaling. Cancer Res. 67, 8439-8443 (2007). 